\section{Significant contribution of intragenic deletions to ARID1B mutation spectrum}

To the Editor:

The arrival of high-throughput sequencing technology has revolutionized the field of medical genetics by steeply increasing the number of identified disease-causing variants, often bringing the previously unknown genes to the center of genetic investigations. The perfect example of this shift is the $A R I D 1 B$ gene, which in just several years became known as one of the most commonly mutated genes in Coffin-Siris syndrome as well as in isolated intellectual disability (ID). The recent work by Sluijs et al. describes the mutational spectrum in 143 patients, including 81 unpublished cases with diseasecausing variants in ARID1B. ${ }^{1}$ Of these, 3 patients carried a large deletion involving neighboring genes in addition to $A R I D 1 B, 9$ patients carried smaller deletions removing one or more exons, and 69 patients had other types of truncating mutations. Even though it was not discussed by the authors, it is important to note that the proportion of intragenic deletions in newly published cases with ARID1B mutations was higher than previously estimated, ${ }^{2}$ as it corresponded to more than $10 \%$. We found similar elevated proportion of intragenic deletions in patients with pathogenic ARID1B variants in our diagnostic centers. We observed six intragenic deletions in 44 previously unpublished cases. Four of these deletions involved a single exon. A previously unreported deletion of exon 9 (NM_020732) was identified in a patient with a phenotype of Coffin-Siris syndrome using gene panel sequencing that included a copy-number variation (CNV) detection method (case 1). Interestingly, the other five intragenic deletions reported in this study were missed during the initial sequencing analysis. In two cases, the initial $A R I D 1 B$ sequencing by a gene panel was returned as normal. Since the clinical presentations of these patients were highly suggestive of Coffin-Siris syndrome, exploration of $A R I D 1 B$ locus was pursued by reanalysis of the obtained sequencing data using a CNV detection algorithm. This approach identified a deletion of exons 1-2 in one patient (case 2) and of exon 16 in another patient (case 3), explaining the phenotypes of these two patients. These intragenic deletions were confirmed by fluorescence in situ hybridization (FISH) and quantitative polymerase chain reaction (qPCR) respectively. In two other cases, the initial exome sequencing was negative, while further $A R I D 1 B$ testing identified deletions removing exon 6 in case 4 and exon 11 in case 5. Exon 6 deletion was identified by gene panel sequencing coupled to a
CNV detection algorithm. It was then confirmed by quantitative multiplex PCR of short fluorescent fragments (QMPSF). Exon 11 deletion was detected by multiplex ligation-dependent probe amplification (MLPA) and then confirmed by gene panel sequencing with a CNV detection step. One deletion involving exons 5-19, initially missed by array comparative genomic hybridization (array-CGH), was identified by gene panel sequencing (case 6).

Diagnostic high-throughput sequencing method consists of two principal steps: sequencing data generation and data analysis. Numerous bioinformatics tools can be incorporated in the data analysis step, or bioinformatics pipeline, potentially leading to a great variability of the results depending on the algorithms used. Considerable international effort has greatly improved and homogenized this process to reduce the discrepancies between results obtained by different diagnostic laboratories. Best practice guidelines are now applied to singlenucleotide variant (SNV) detection approaches to standardize the bioinformatics analysis. No such guidelines are currently available for $\mathrm{CNV}$ detection in high-throughput sequencing data, despite the existence of multiple different algorithms. Indeed, screening the sequencing results for presence of deletions or duplications is still not systematic in certain diagnostic centers. There is growing evidence that CNVs represent a substantial proportion of pathogenic variation in different types of pathologies. They are especially common in neurological diseases. ${ }^{3}$ Given the clinical importance of CNVs, many diagnostic laboratories, including ours, are using the $\mathrm{CNV}$ detection algorithms in the analysis pipeline to process the data obtained by high-throughput sequencing. Since ARID1B is now present in many gene panels worldwide, it is not surprising that the number of intragenic deletions identified in this gene continues to rise. Thus, it is likely that the contribution of intragenic deletions to the $A R I D 1 B$ mutation spectrum will be even higher once CNV detection methods become more efficient and more systematically applied.

In three cases described here, array-CGH did not identify the disease-causing intragenic deletion. In case 6 , an $8 \times 60 \mathrm{~K}$ array-CGH (Agilent) with the resolution fixed at $400 \mathrm{~kb}$ was performed in a prenatal setting, failing to identify the deletion involving exons 5-19 of ARID1B. Deletions in cases 2 and 3 were missed by array-CGH $8 \times 60 \mathrm{~K}$ and $4 \times 180 \mathrm{~K}$ (Agilent) respectively due to insufficient resolution. To further explore the reasons for these false negative results, we carefully examined the CGH probe coverage of the ARIDIB locus. Strikingly, we found that a $75-\mathrm{kb}$ region containing the last 12 exons of this gene (exons 9-20) was not covered by CGH probes for the arrays most often used in diagnostics (e.g., Agilent $4 \times 180 \mathrm{~K})$. Even though this region is detectable by several high-resolution arrays, these methods are rarely used in routine diagnostics due to elevated cost and increased 
interpretation time. Thus, it is possible that other patients carrying small intragenic deletions in the 3' region of $A R I D 1 B$ gene might have received a false negative result following a routine array-CGH. It is important to note that a deletion of any single exon in this region (exons 9-20) will lead to frame shifting, with the exception of exon 12 removal. Thus, even small deletions involving single exons in the 3' part of the gene will likely have a loss-of-function effect on ARID1B protein.

Our findings complete the $A R I D 1 B$ mutation spectrum described by Sluijs et al. by providing valuable information about intragenic deletions in this gene. Based on the data from their 81 cases and our additional 44 unpublished cases with pathogenic variants in $A R I D 1 B$, we conclude that intragenic deletions play an important role in the mutation spectrum of this gene. Our experience also stresses the importance of clinicians' input during diagnostic sequencing interpretation, since in most of our cases reanalysis of ARID1B gene was based on the high suspicion of Coffin-Siris syndrome. Given the frequency of intragenic deletions in $A R I D 1 B, \mathrm{CNV}$ detection at this locus will increase the diagnostic rate and in certain cases will help bring long-awaited diagnoses to patients.

\section{DISCLOSURE}

The authors declare no conflicts of interest.

Publisher's note: Springer Nature remains neutral with regard to jurisdictional claims in published maps and institutional affiliations.

Svetlana Gorokhova, $M D, P h D$ (D) $^{1,2}$, Jeremie Mortreux, $M D$ (D) $^{1,2}$, Alexandra Afenjar, $M D^{3,4,5}$, Tania Attie-Bitach, $M D, P h D$ iD $^{6,7,8}$,
Maud Blanluet, PharmD 9 , Valérie Cormier-Daire, $M D, P h D^{6,7,10}$, Anne-Marie Guerrot, $M D^{11}$, Anne-Sophie Lebre, PharmD, $\mathrm{PhD}^{12}$, Valérie Malan, $M D, P h D^{6,7,8}$, Gael Nicolas, $M D, P h D$ (D) ${ }^{11}$, Sophie Rondeau, PharmD ${ }^{6,7,13}$, Nicole Philip, $M D^{1,2}$,

Pascale Saugier-Veber, PharmD, PhD ${ }^{11}$, Catherine Badens, PharmD, $P h D^{1,2}$ and Chantal Missirian, $M D^{1,2}$

${ }^{1}$ Département de Génétique Médicale, APHM, CHU Timone Enfants, Marseille, France; ${ }^{2}$ Aix Marseille University, MMG, INSERM, Marseille, France; ${ }^{3}$ Département de génétique et embryologie médicale, Hôpital Trousseau, Assistance publique-Hôpitaux de Paris, Paris, France; ${ }^{4}$ Centre de Référence malformations et maladies congénitales du cervelet et Déficience Intellectuelle de Causes Rares, Paris, France; ${ }^{5}$ Sorbonne Universités, GRC ConCer-LD, Hôpital Armand Trousseau, Paris, France; ${ }^{6}$ Fédération de Génétique Médicale, Université Paris Descartes-Sorbonne Paris Cité, Paris, France; ${ }^{7}$ INSERM UMR1163, IMAGINE Institute, Paris, France; ${ }^{8}$ Service d'histologieembryologie-cytogénétique, AP-HP, Hôpital Necker-Enfants Malades, Paris, France;

${ }^{9}$ Department of Genetics, Institut Curie, Paris, France; ${ }^{10}$ Service de Génétique Clinique, Hôpital Necker-Enfants Malades, AP-HP, Paris, France; ${ }^{11}$ Normandie Univ, UNIROUEN, Inserm U1245 and Rouen University Hospital, Department of Genetics and Reference Center for Developmental Disorders, F 76000, Normandy Center for Genomic and Personalized Medicine, Rouen, France; ${ }^{12}$ Department of Genetics, Reims University Hospital, Reims, France; ${ }^{13}$ Service de Génétique Moléculaire, Hôpital Necker-Enfants Malades, AP-HP, Paris, France. Correspondence: Svetlana Gorokhova (svetlana.gorokhova@univ-amu.fr)

\section{REFERENCES}

1. van der Sluijs PJ, Jansen S, Vergano SA, et al. The ARID1B spectrum in 143 patients: from nonsyndromic intellectual disability to Coffin-Siris syndrome. Genet Med. 2018. https://doi.org/10.1038/s41436-018-0330z [Epub ahead of print].

2. Schrier Vergano S., et al. Coffin-Siris syndrome. In: Adam MP, et al., (Eds.) GeneReviews. Seattle, WA: University of Washington; 2018.

3. Truty R, Joshua P, Kennemer M, Lincoln SE, Olivares E, Nussbaum RL, Aradhya $S$. Prevalence and properties of intragenic copy-number variation in Mendelian disease genes. Genet Med. 2019;21:114-123.

Advance online publication 20 May 2019. doi:10.1038/s41436-019-0546-6 